\title{
Technology and Market Conditions Toward a New Competitive Landscape in the Wireless Access Market
}

\author{
Fernando Beltrán, The University of Auckland \\ Jairo A. Gutiérrez, Universidad Tecnológica de Bolívar \\ José Luis Melús, Polytechnic University of Catalonia
}

\begin{abstract}
This article argues that the wireless telecommunication market is about to witness a shift in business models and market structure as a result of the deployment of new broadband access technologies, spectrum management techniques, policy-based network management, and the drive of new entrants to compete against the incumbents. The article discusses four agents of evolutionary changes: a range of broadband wireless access standards and technologies that are frontrunners in the industry's efforts to embody the next generation of wireless networks; new provider-customer relationships facilitated through changes in the usual contract patterns that will allow consumers to enter short-term and spot contracts with the new wireless providers; an overview of the current debate on spectrum management; and an explanation of how autonomic communications and policybased management would support the new structure. Finally, the article asserts the necessity for the integration of the heterogeneous technologies that make up this emerging, hybrid wireless landscape, and describes the economic characteristics of a new competitive scenario.
\end{abstract}

\section{INTRODUCTION}

Amid the global economic recession at the end of the first decade of the 21st century, wireless telecommunications and related industries remain one of the most successful and profitable markets worldwide. Industry estimates indicate that cellular subscribers around the world will pass the 4 billion mark, with demand picking up in 2010 and sales growing by 7 percent, even though sales of mobile devices to end users decreased 3.7 percent in 2009 [1]. Forecasts also indicate a steady growth during 2011 to 2013 in the global mobile infrastructure market, driven mainly by emerging market growth and a shift toward wideband code-division multiple access (WCDMA) and Long Term Evolution (LTE) technologies [2].
Cellular companies have been able to expand the range of services they provide, which originally only consisted of voice, directory, and other minor information services, to a large range of new services that are now supported by their third generation $(3 G)$ and soon, their fourth generation $(4 \mathrm{G})$ networks. With their firm grip on customers, cellular providers have been able to successfully squeeze user's value from a range of new mobile services. But it is not only the direct provision of services that makes them succeed in the marketplace; roaming charges seem unjustifiably large with companies relying on the inconveniences and difficulties associated with changing providers for a short period of time (say, while traveling abroad) to retain their customers. Mobile telecommunications providers seek not only to keep but also to strengthen the long-term commercial relations with their customers. Their proprietary access technologies serve their business plans even when users have to carry two different phones or two different access cards to be able to use different services from different providers.

Clearly the shift of the industry to the next generation of mobile networks requires innovative business models to satisfy the increasing demand for mobile broadband access and Internet applications and services. This shift will offer consumers not only higher data rates and more efficient systems, but also broadband Internet access able to support innovative multimedia services and applications. More important, there is growing awareness that these goals would be achieved if interoperability and roaming functions for heterogeneous terminals across different networks are to be assured [2]. In recent years the deployment of IP-based services, such as Skype, over unlicensed wireless hot-spots has diminished the barriers for new actors who can now compete for a market share of the wireless access business. These low-cost network access solutions allow these new entrants to establish agreements with similar counterparts in order to extend their services. Table 1 illustrates the range and scope of emerging mobile services. 
Mobile financial services

Mobile advertising

Mobile tracking

Product location and shopping assistant services

Mobile entertainment services and games

Mobile distance education

Wireless data center

Time-critical mobile services

Ubiquitous communication
Banking, stock brokerage, and payments for mobile users

Location-based services

Location tracking of goods, boxes, and people (troops, refugees, children)

Finding the location of particular products, cost-comparison services

Video-on-demand, audio-on-demand, interactive gaming

Audio and video streaming of classes

Download of product information by vendors and sellers

Personal medical services; notification, reminder, and alert service; airline schedule information

Mobile voice communication, SMS, e-mail, video mail
Open

Both

Open

Open

Both

Closed

Closed

Both

Both

*Typical configuration

Table 1. Classes of mobile services (based on [3]).

The third column has an indication of the type of implementation for those services with closed (or walled-garden) configurations favored by incumbent operators, while open services, in the tradition of the Internet, are promoted as a competitive advantage by emerging operators.

In this article we describe an evolutionary path that suggests how access to mobile telecommunications will unfold. We argue that new technologies, (i.e., software agents, policy-based network management, autonomic computing techniques, and cognitive radio) constitute a promising platform for new entrants to the mobile arena to offer services differently. In combination with dynamic pricing and spectrum allocation schemes, these technologies offer alternatives to locked-in contractual relationships between provider and customer. The path suggested here is one in which access options are increased, possibly attracting agile service providers with the potential to collectively reshape the wireless network services markets. These new entrants to the marketplace will be able to challenge the incumbent telecommunication companies while offering customers a competitive option.

In the next section we will discuss a range of broadband wireless access standards and technologies that are the front-runners in the industry's efforts to provide next-generation networks and the new services they would be able to support. We then provide an overview of the current debate on spectrum management, a crucial issue in the development of new wireless broadband access. We also discuss a key technological development that will help settle the spectrum debate, namely, cognitive radio networks. We then introduce the concepts of autonomic communications and policy-based management, as well as posing elements foreshadowing the integration of the heterogeneous technologies that make up the emerging hybrid, competitive wireless landscape. We then argue that, contrary to traditional business arrangements by cellular operators, consumers will be able to enter shortterm and spot-contracts with the new wireless providers. Before the concluding remarks, the final section discusses some economic characteristics of the new competitive scenario.

\section{BROADBAND WIRELESS ACCESS TECHNOLOGIES}

Alternatives to $3 \mathrm{G}$ cellular technologies (such as CDMA 2000 and Universal Mobile Telecommunications Systems [UMTS]) are being announced as the next step in the evolution of mobile communications technologies. The most important forerunners are WiMAX, the IEEE 802.20 standard (Mobile Broadband Wireless Access [MBWA]), and the 4G of mobile communications, also known as LTE.

Standardization bodies such as the WiMAX Forum are working to unveil a new standard that will offer $100 \mathrm{Mb} / \mathrm{s}$ to enable mobility and $1 \mathrm{~Gb} / \mathrm{s}$ for fixed applications, as early as 2012 . MBWA seeks the deployment of spectrum efficient, ubiquitous, always-on, and interoperable mobile broadband wireless access. This is addressed to end-user markets that include access to Internet, intranet, and enterprise applications by mobile users and access to information and entertainment sources. At the same time the telephony world is preparing their current $3 \mathrm{G}$ networks for the transition to LTE driven by proposals to reach $300 \mathrm{Mb} / \mathrm{s}$ downstream and $100 \mathrm{Mb} / \mathrm{s}$ upstream. With such fast rates they promise the provision of television, telephony, and Internet services. Table 2 summarizes the main broadband wireless access technologies discussed in this section.

Wireless operators will have the opportunity to deploy systems that can achieve very high rates and aggregate data capacity within a mini- 


The main players in
the emerging
competitive
landscape belong to
two main camps:
cellular networks
transitioning towards
and using LTE,
on one side, and a
competitive fringe
composed of
multiple, more
numerous providers.

\begin{tabular}{|c|c|c|c|c|c|}
\hline Characteristics & $\begin{array}{l}802.1 \\
\text { WiFi }(802.11 \mathrm{~g})\end{array}$ & $\begin{array}{l}802.16 \text { e } \\
\text { Mobile WiMAX }\end{array}$ & $\begin{array}{l}802.20 \\
\text { MobileFi }\end{array}$ & $3 G$ & LTE \\
\hline Bands & $2.4 \mathrm{GHz}$ & $2-6 \mathrm{GHz}$ & $<3.5 \mathrm{GHz}$ & $<2.7 \mathrm{GHz}$ & $<5 \mathrm{GHz}$ \\
\hline Channel bandwidth & & $>5 \mathrm{MHz}$ & $<5 \mathrm{MHz}$ & $<5 \mathrm{MHz}$ & $<20 \mathrm{MHz}$ \\
\hline Maximum data rate & $<54 \mathrm{Mb} / \mathrm{s}$ & $<70 \mathrm{Mb} / \mathrm{s}$ & $\begin{array}{l}5 \mathrm{MHz}, 120 \mathrm{~km} / \mathrm{h} \\
5 \mathrm{Mb} / \mathrm{s} / \mathrm{sect} .(\mathrm{D})\end{array}$ & $3 \mathrm{Mb} / \mathrm{s}$ & $\begin{array}{l}300 \mathrm{Mb} / \mathrm{s}(\mathrm{D}) \\
100 \mathrm{Mb} / \mathrm{s}(\mathrm{U})\end{array}$ \\
\hline Maximum cell range & $100 \mathrm{~m}$ & $50 \mathrm{~km}$ & $>15 \mathrm{~km}$ & $5 \mathrm{~km}$ & $<30 \mathrm{~km}$ \\
\hline
\end{tabular}

Table 2. Broadband wireless access technologies.

mum amount of spectrum. To achieve this they will have to reduce the number of cell sites required in the network for such enhanced performance characteristics. That implies that the cost of deploying and operating 802.20 networks will be reduced and consumers will have access to higher speed data services at more affordable prices. The IEEE 802.20 standard will fill the gap between cellular networks, which exhibit low bandwidth and high mobility, and the WiFi (IEEE 802.11) standard for wireless networks, with high bandwidth and low mobility, and will provide seamless integration among work, home, and mobile usage, offering transparent support of real-time and non real-time applications. Further enhancements associated with WiMAX networks have led to the specification and development of yet another IEEE standard: 802.22, which defines wireless regional area networks (WRANs) and uses large cells (radios of approx. $100 \mathrm{~km}$ ) to provide coverage in remote and unpopulated areas

Other proposals aligned with the existing $3 \mathrm{G}$ cellular technologies, which together with the UMTS are predominant in the market, plan to establish themselves as the preferred option for future access technologies. A significant development in this area is Ultra Mobile Broadband (UMB), which is based on TCP/IP, builds on CDMA 2000, and offers rates of up to $280 \mathrm{Mb} / \mathrm{s}$.

Figure 1 illustrates an emerging hybrid situation, which is likely to arise when overlapping wireless technologies are deployed in an urban area. Such a hybrid situation should be able to support unimode terminal access, such as when multimode terminals access Wireless Local Area Network (WLAN) access-enabled equipment, which is also configured with a cellular interface. The main players in the emerging competitive landscape belong to two main camps: cellular networks transitioning toward and using LTE, on one side, and a competitive fringe composed of multiple, more numerous providers. This latter group will operate on the array of technologies being standardized by the IEEE 802 working groups and which include WiFi, WiMAX, and WRAN configurations, while the incumbents will support their current evolution plans toward 4G/LTE networks.

\section{SPECtRUM MANAGEMENT}

Current spectrum allocation policies for wireless networks are characterized by a limited, static spectrum that regulatory agencies allocate to licensed holders on a long-term basis for large geographical or densely populated regions. In recent years the generalized use of the Internet Protocol (IP) together with the increasing presence of unlicensed wireless network access, for instance WLANs, has reduced some of the entry barriers to new operators, including inflexibility, cost, and inefficiency of traditional spectrum allocation mechanisms. Furthermore, recent mobile broadband developments provide opportunities for those new operators, with mobility as the key driving force. Consequently, the presence of new operators translates into an increasing demand for spectrum; nevertheless, such increase renders obsolete the application of the current spectrum allocation policy, in particular for some spectrum bands.

How spectrum governance should be improved is being debated in many countries. The debate focuses on the potential of a new spectrum policy to alleviate an already diagnosed artificial spectrum scarcity, promote efficiency, and encourage innovation. Two distinct visions are present in the debate: spectrum property rights and spectrum commons [4]. According to the spectrum property rights perspective, market forces determine the allocation of spectrum and those who obtain it, own it. Holders can also use the spectrum for any purpose, including the possibility of trading in secondary markets. The current approach to spectrum policy, which partially implements the property rights scheme, is to license the use of spectrum. Nevertheless, as technology evolves, the feasibility of the spectrum commons approach becomes evident. Spectrum commons advocates for the shared use of spectrum, with a common access regime or protocol. Technologies able to avoid interference and provide seemingly non-scarce spectrum availability are known as smart technologies. Devices endowed with smart technologies will be able to negotiate spectrum band sharing. Hence, the spectrum commons approach envisions multiple providers or access points efficiently sharing spectrum bands with no interference and a minimally enforced control protocol.

As a large portion of the assigned spectrum is used sporadically, leading to underutilization of significant amounts of spectrum [5], significant advances have been achieved in the dynamic use of spectrum by new high-speed access technologies. One key enabling technology of dynamic spectrum access is cognitive radio (CR), which provides the capability to share the wireless channel with licensed users in an opportunistic 
manner [6]. Cognitive networks, or CR-based networks, are reconfigurable wireless networks that adapt to all types of circumstances by means of self-management. Cognitive networks are becoming the technology factor that could facilitate the transition to a more competitive mobile telecommunication landscape [7]. With cognitive wireless networks, licensed users can offer dynamic sharing spectrum opportunities to unlicensed users or service providers. Such an arrangement would be the facilitator of new access opportunities to secondary (unlicensed) providers, increasing the efficiency in spectrum utilization. CR networks are envisioned to provide high bandwidth to mobile users via heterogeneous wireless architectures and dynamic spectrum access techniques.

\section{INTEGRATION OF Heterogeneous Networks as a COMMON Business PlatForm}

The success of cognitive networks lies in their capability to continuously adapt to varying environmental conditions and users' needs. Their most prominent feature, that is, their self-management functionality, is associated with the autonomic communications management paradigm [8]. The essence of autonomic management is the capability of a network to selfgovern its behavior. Their management functionality must take into account the context within which communication occurs; in other words, management has to be context-aware. In wireless communications the context includes key aspects such as coverage, power conditions in the handset, traveling speed, and surrounding features, which may be important to enable location-based services. In addition to context, it must also factor in the policies put in place by operators, the experience and knowledge of the user, and the plausibly conflicting goals pursued by both parties.

Autonomic communications reduce the complexity associated with managing large-scale communications networks. Thus, their aim is to simplify network management processes by automating and distributing the decision-making processes involved in optimizing network operations. A novel autonomic architecture could dynamically synthesize the context knowledge, relevant aspects of the environment, and other characteristics (capabilities, constraints, etc.) in order to adapt its network functionality to current conditions. A network endowed with autonomic management needs to address the resource allocation problem within the constraints of the business rules imposed on it. It also must own the means to learn and interpret changes in the surrounding environment; many authors refer to such means as policies, and their implementation as Policy-Based Management (PBM). PBM proposes information and ontological modeling to capture knowledge relating to network capabilities, environmental constraints, and business goals and policies, together with reasoning and learning techniques, to enhance and evolve such knowledge [9].

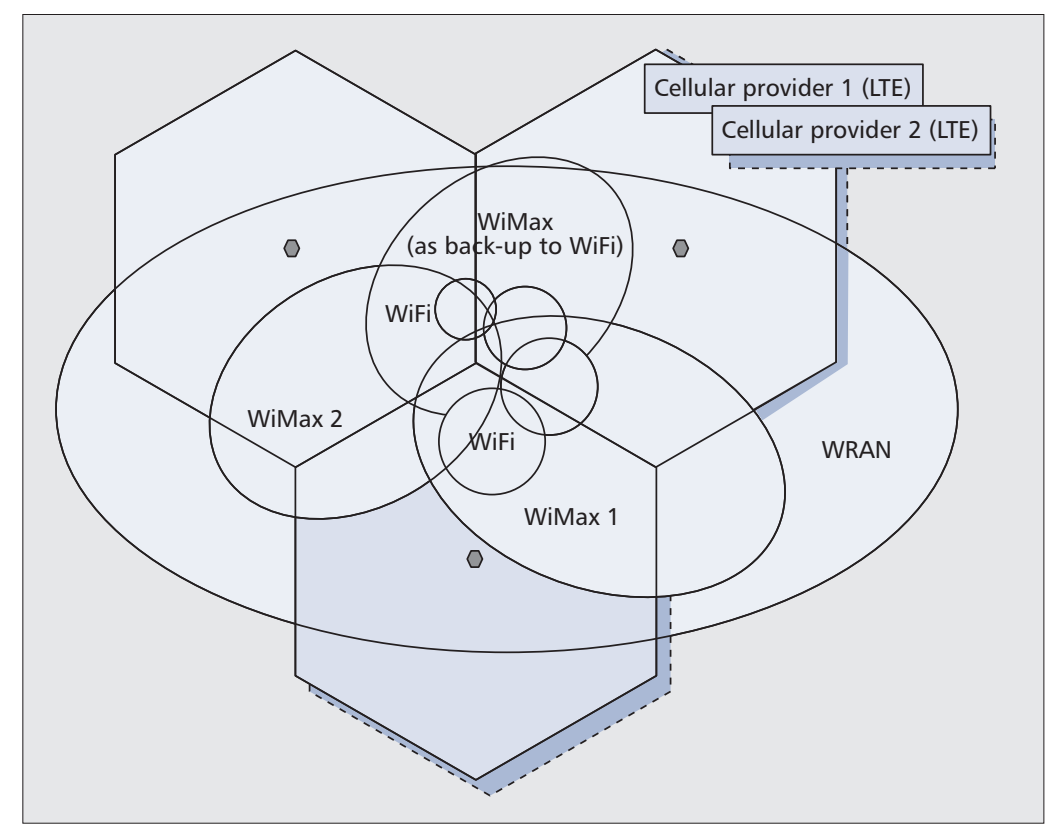

Figure 1. A heterogeneous broadband wireless access landscape.

The PBM approach is focused on providing support that makes it possible to automatically assign network resources, control quality of service (QoS), and monitor resources, using a set of previously defined policies. A policy is a directive that manages certain aspects of the behavior of the system, and it depends on the interaction of users, services, and existing resources. The implementation of the policies is based on the basic rule, "if event and conditions then actions." The flexibility of the PBM paradigm makes it a first candidate to service management in a dynamic and distributed context, not only because of the number of possibilities that could be opened but also because of their diversity. The infrastructure so developed would allow the creation of a methodology that could be considered as one of the key enablers of future autonomic communications. Most of the work in the PBM research field has focused on creating an automatic generation model for service management policies that deals with a variety of devices and services, developing formal methods to allow policy conflict detection and policy resolution.

In the scenario developed in this article, the policies should be automatically generated without intervention of manual operators and derived directly from the requirements of the users, the services, and the devices. Requirements, also known as policy generation objectives, could change so that the patterns suggested by such changes could be used to model dynamic behavior. However when such capabilities get to be deployed on seemingly disparate wireless access technologies, network operators would need to address yet another issue that would facilitate the introduction of new business models: the integration and management of heterogeneous wireless access technologies.

Integrating heterogeneous wireless networks would enable a multimode wireless device equipped with multiple interfaces to use appro- 


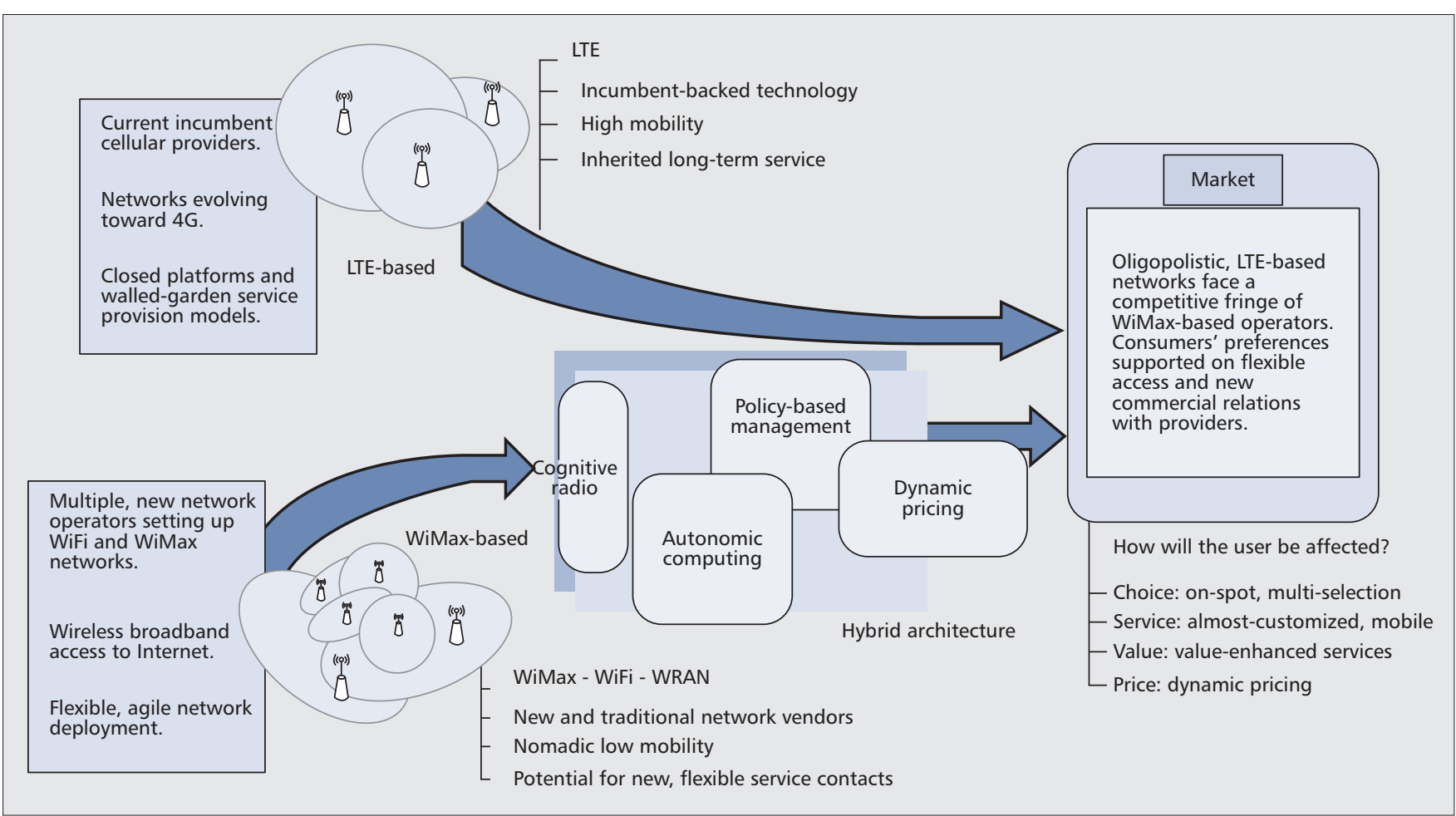

Figure 2. Broadband access wireless markets and a proposed path of market evolution.

priate networks that meet their service and cost requirements. The wireless communications of the future will have to integrate multiple wireless access technologies in a seamless manner. In such a heterogeneous environment, the efficient allocation of resources becomes even more critical for success.

Heterogeneous wireless access networks should facilitate the provision of seamless services to the end-user and freedom of movement between different geographical areas. The network management systems that currently support these facilities need to be improved to obtain a new architecture, which would enable users to accomplish their tasks independently of the device being used, the chosen media, or the underlying technology. In our view the most important network management development that can address those issues are the techniques and standards associated with PBM. They include a well-structured approach to policy rules and policy applications, crucial when dealing with large and complex networks, and a key to the efficiency and scalability of the policy conflict analysis, used to cope with the dynamical aspects of such communications networks.

Figure 2 illustrates an evolving scenario that integrates main access technologies involved and their competitive features. On the competitive fringe occupied by new entrants, the interface between the access layer technologies is clearly separated from other enabling technologies. Dynamic pricing, considered here as a practice that supports the pricing strategy of the competitive fringe, is possible due to the interaction between autonomic computing on top of cognitive radio network deployments, on one hand, and the utilization of policy-base management on the other. Those system components are clearly intermingled with no easy functional separation as with a traditional layer model. The key technologies mentioned here can potentially facilitate the adoption of dynamic prices, which would empower innovative competitors and enable them to develop successful business models in the wireless arena.

\section{SHORT-TERM CONTRACTS ENABLED BY NetWork MANAgEMENT TECHNIQUES}

The battle for technological supremacy in the next generation of wireless networks has two main players: WiMAX and LTE. Industry experts in one camp gathered at recent versions of the World Mobile Congress in Barcelona, Spain claim that "LTE is still in its very early stages" and forecast the adoption of LTE to be commercialized in the $\mathrm{mid} /$ long term. Industry representatives in the other camp assert that: "the current economic conditions may give WiMAX more foothold in the market in the longer term." Such announcements seem to indicate that WiMAX and LTE are seen as battling for future consumers of mobile services.

WiMAX is being embraced by newcomers who hope to become established first in the mobile Internet access business, while LTE, endorsed mainly by leading wireless telephony companies, is being built on existing infrastructure, and the companies expect to see first deployments working as of 2015.

The WiMAX Forum reported more than 559 
WiMAX networks in 147 countries in the first quarter of 2010, and forecasts worldwide coverage to nearly 800 million people by the end of 2010. Newcomers, pushing their way into the market, are giving WiMAX a go while deploying urban and suburban access networks. In Russia, Scartel operates a Mobile WiMAX network; in the U.S., Sprint announced WiMAX deployment in 10 major U.S. cities in 2010 after its successful network launch in Baltimore, while Digital Bridge has launched WiMAX services in Jackson, Wyoming, and Ashburn, Virginia; Max Telecom, in Bulgaria, has rolled out a mobile WiMAX, providing voice and MaxTV services; in 2008 a joint venture between Bell Canada and Rogers communications launched the first phase of a future countrywide WiMAX network in Canada; in 2007, Germany Alcatel Lucent built a WiMAX net for VSE Net, while more recently Singapore's QMAX communications launched a mobile WiMAX service with major coverage in a $15 \mathrm{~km}$-radius area.

The current market structure in the mobile area is an oligopoly, that is, a market with very few competitors, each of which has captured an important share of the market. Oligopolistic competition is not strong and is further handicapped by the operators' strategic need to keep their customers locked in. Lock-in practices include service contracts with minimum-time or earlier disconnection penalties. Network convergence, realized through LTE, would allow traditional operators to increase their service platform and be able to increase their bundlingservice ability, which exemplifies yet another, more suitable locked-in effect.

As WiMAX networks are deployed, sometimes also providing back-haul service to WiFi hotspots, such hybrid network arrangements begin to erect themselves as alternatives to the new mobile LTE deployments. Contrary to the non-open, proprietary characteristics of mobile networks, WiMAX operators might find it to their advantage to cooperate, therefore offering users' devices a common language that devices and access points can use. The latter is not to suggest a clear-cut, closed vs. open paradigm brought about by LTE and WiMAX, respectively; it simply highlights the potential a competitive fringe of operators may develop in order to compete while maintaining a common access language to their access infrastructures.

As some customers may dislike long-term commitments to service providers and would prefer to do business with any provider capable of accommodating their demand on a very shortterm basis, new providers might capture such preference in their business plans. New providers therefore might innovate on the way service contracts have traditionally been offered, and might explore the feasibility of providing connection-as-you-go or spot contracts. Pre-paid cellular access plans can be considered a precursor to these more flexible and possibly more attractive commercial relations.

The adequate establishment of policy rules, when using PBM mechanisms can enable the dynamic allocation of prices to the services requested by the users [10]. In the mixed sce- nario that we have described, a key factor in price setting is the context within which the services are demanded. Thus, providers will find it necessary to use pricing models that take into account the service context and particularly the availability of resources. Moreover, pricing mechanisms must consider the way to keep users informed about the evolution of current prices. When price is based on flat rates or static schemes that do not represent the current context within which services are requested, an imbalance is created between providers and users. Such a situation does not stimulate improvements in the quality of the services or the creation of a clear competitive market that leads to significant reduction in prices for these services. PBM can therefore be used as a promoter of dynamic price allocation and a facilitator of short-term contracts.

\section{A NeW Competitive Landscape}

Current market structure and the conditions of retail mobile service contracts strengthen the incentives mobile operators have to maintain their locked-in service offers, while finding in next-generation wireless networks, particularly LTE, the source of further cost reductions and the promise of service and network convergence. Customers' expectations will be realized through service offerings that not only include traditional voice services but also video-on-demand, TV service, Internet access, and others. On the other hand, as new and traditional providers push their way into the access market, customers may now realize that WiMAX providers and the hybrid networks that will derive could erect themselves as alternatives to those new mobile LTE deployments.

Having to face and compete against the market power of the incumbent mobile operators, newcomers would be pressed to find cooperative ways to break through the marketplace. Standardization of the short-term access contract is a possible path. If new operators managed to agree on a standardized way for users' devices to treat the pricing and condition information about their service plans, users would not have to worry about price comparisons, price search, better deals, and the like. Such a scenario is going to demand serious thought by the new providers who would certainly benefit to the extent that they can successfully challenge the established operators and their new technology. A customer would only need a few key strokes here and there to describe their requirements to a software agent. Such information would delineate a consumption profile in which preferred services, willingness to pay, and preferred call destinations, among others, would be used by the agent to decide which access network connection is best.

Pricing rules should be developed over open platforms that guarantee not only interoperability among the providers, which would facilitate their cooperation, but also the implementation of their individual business strategies. This dynamic mobile market scenario offers an enormous potential for the adoption of short-term customer-provider relations.

\section{Pricing rules should \\ be developed over open platforms that guarantee, not only the interoperability among the providers, which would facilitate their cooperation, but the implementation of their individual business strategies.}


As the market for

broadband wireless

access witnesses the

entry of new urban

and suburban

providers, our article

suggests that if

newcomers are able

to change the nature

of the customer-

provider relationship,

they will successfully

challenge the

incumbents'

wireless oligopoly.

\section{CONCLUSIONS}

The market landscape suggested in this article is fueled by the competing presence of two independently developed technology camps: LTE, representing the next-generation of mobile cellular networks, and WiMAX, a wireless broadband access technology with mobility limitations. Recent advances in WiMAX and related hybrid networks bring with them the possibility of shifting customers' perceptions toward the realization of a substitute access technology that can partially bridge the mobility gap while providing access to a broad range of mobile services. We suggest that as the market for broadband wireless access witnesses the entry of new urban and suburban providers, if newcomers are able to change the nature of the customer-provider relationship, they will successfully challenge the incumbents' wireless oligopoly. This article envisions the new operators acting as a competitive fringe that collectively adopts enabling techniques, such as cognitive networks, policy-based network management, and autonomic computing, in order to seamlessly provide customers with more flexible, dynamic pricing service arrangements.

The range of broadband wireless access technologies is very promising and will certainly attract both customers and providers to shortterm relationships for service provision. Not being able to reclaim its supremacy in the mobile access markets, the contending camp's traditional service contract may significantly change. As suggested here, such is the nature of the new landscape of the wireless access market.

\section{REFERENCES}

[1] Gartner, "Forecast: Mobile Infrastructure, Worldwide, 2004-2013," Sept. 2009.

[2] K. Etemad, "Overview of Mobile WiMAX Technology and Evolution," IEEE Commun. Mag., vol. 46, no. 10, 2008, pp. 31-40.

[3] U. Varshney and R. Vetter, "Mobile Commerce: Framework, Applications, and Networking Support," Mobile Net. Apps., vol. 7, no. 3, 2002, pp. 185-98.

[4] J. Brito, "The Spectrum Commons in Theory and Practice," Stanford Tech. Law Rev. 1, 2007.
[5] O. Ileri and N. B. Mandayam, "Dynamic Spectrum Access Models: Towards an Engineering Perspective in the Spectrum Debate," IEEE Commun. Mag., vol. 46, no. 1,2008 , pp. 153-60

[6] I. F. Akyildiz et al., "A Survey on Spectrum Management in Cognitive Radio Networks," IEEE Commun. Mag., vol. 46 , no. 4, 2008, pp. 40-48.

[7] B. Xie, A. Kumar, and D. P. Agrawal, "Enabling Multiservice on $3 \mathrm{G}$ and Beyond: Challenges and Future Directions," IEEE Wireless Commun., June 2008, pp. 66-72.

[8] K. Demestichas et al., "Towards Cognitive B3G Networks: Autonomic Management of Access Points," 16th IST Mobile Wireless Commun. Summit '07, 2007.

[9] B. Jennings et al., "Towards Autonomic Management of Communications Networks," IEEE Commun. Mag., vol. 45 , no. 10, 2007, pp. 112-21.

[10] D. Niyato and E. Hossain, "Competitive Pricing in Heterogeneous Wireless Access Networks: Issues and Approaches," IEEE Network, Nov./Dec. 2008, pp. 4-11.

\section{BIOGRAPHIES}

FERNANDO BELTRAN (f.beltran@auckland.ac.nz) is currently a Senior Lecturer in the Information Systems and Operations Management Department, University of Auckland Business School, New Zealand, where he leads the Pricing in NextGeneration Networks (PING) research group. He received a B.S. degree in Electrical Engineering from the Universidad de Los Andes, Bogotá, Colombia, and a Ph.D. in Applied Mathematics from SUNY, Stony Brook, NY. His research focuses on pricing in Next-Generation networks. He has been a visiting scholar at the FCC and the CITI Columbia Business School.

JAIRO A. GUtiéRREZ (jgutierrezd@unitecnologica.edu.co) is a Full Professor with the Systems Engineering Department, Universidad Tecnológica de Bolívar, Cartagena, Colombia. He has recently been with the Information Systems Department at the University of Auckland, and was the the Editor-in-Chief of the International Journal of Business Data Communications and Networking (2004-2008). He received his Systems and Engineering degree from the Universidad de Los Andes, a Masters degree in Computer Science from Texas A\&M University, and a Ph.D. in Information Systems from the University of Auckland. His current research is on network management systems, viable business models for mobile commerce, and quality of service in Internet protocols.

JosÉ LUIS MeLUs (jlmelus@entel.upc.edu) is a Full Professor at the Polytechnic University of Catalonia in the Telecommunications Engineering Department. He holds both a B.S. degree and a Ph.D. degree in telecommunications from Escuela Técnica Superior de Ingenieros de Telecomunicación, Barcelona, Spain. His present research interests include network management, quality of service, planning and evaluation of networks of computers, and security and pricing telecommunications services. 